\title{
DIVERSIDAD DE MACROINVERTEBRADOS ASOCIADOS A ARRECIFES DE PHRAGMATOPOMA MOERCHI KINBERG, 1867 (POLYCHAETA: SABELLARIIDAE) EN EL INTERMAREAL ROCOSO DE COCHOLGÜE, CHILE
}

\author{
MACROINVERTEBRATE DIVERSITY ASSOCIATED TO REEFS OF \\ PHRAGMATOPOMA MOERCHI KINBERG, 1867 (POLYCHAETA: \\ SABELLARIIDAE) IN THE INTERTIDAL ROCKY SHORE AT COCHOLGÜE, CHILE
}

\author{
Roger D. Sepúlveda ${ }^{1,2}$, Rodrigo A. Moreno ${ }^{2,3}$ \& Franklin D. Carrasco ${ }^{4}$ \\ ${ }^{1}$ Departamento de Ecología Costera, Facultad de Ciencias, Universidad Católica de la Santísima Concepción, Casilla \\ 297, Concepción, Chile. \\ ${ }^{2}$ Programa Magíster en Zoología, Universidad de Concepción. \\ ${ }^{3}$ Departamento de Ciencias del Mar, Universidad Arturo Prat, Casilla 121, Iquique. \\ ${ }^{4}$ Departamento de Oceanografía, Facultad de Ciencias Naturales y Oceanográficas, Universidad de Concepción, Casilla \\ 160-C, Concepción, Chile. E-mail: rogers@ucsc.cl
}

\section{RESUMEN}

Las agregaciones monoespecíficas son comunes en el intermareal rocoso. Una de estas especies gregarias es el poliqueto tubícola Phragmatopoma moerchi, el cual construye arrecifes de tamaño variable en el intermareal rocoso. Estos arrecifes proveen de refugio y alimento a muchas especies de invertebrados. El objetivo de este trabajo es caracterizar la macrofauna asociada a estos arrecifes y determinar cómo afecta el tamaño de éstos a algunos parámetros comunitarios. Nueve muestras fueron extraídas del intermareal de Cocholgüe ( $36^{\circ} 35^{\prime} \mathrm{S}$ ), entre octubre y diciembre de 2001. En laboratorio, las muestras fueron pesadas y la fauna asociada fue agrupada por taxa y determinada, cuando fue posible, al nivel taxonómico más bajo posible. Luego se obtuvieron los descriptores comunitarios (número de individuos, diversidad, riqueza, dominancia y uniformidad) para relacionarlos con el tamaño de los arrecifes (TA). Fueron encontrados un total de 487 especímenes de 43 taxa pertenecientes a nueve grupos mayores. Los taxa con más especies presentes fueron Mollusca y Polychaeta (17 y 10 , respectivamente). Relaciones directas y significativas fueron encontradas entre el TA y algunos descriptores comunitarios; los valores de diversidad fueron altos, pero no significativamente correlacionados con el TA. Los resultados del presente estudio demuestran que estos arrecifes constituyen un microhábitat que alberga una gran cantidad y diversidad de invertebrados, y por lo tanto, podrían ser importantes para mantener la biodiversidad local.

Palabras ClaVes: Intermareal rocoso, macrofauna, biodiversidad, Polychaeta, Phragmatopoma moerchi, arrecifes.

\section{ABSTRACT}

Single species aggregations are common in the rocky intertidal shore. One of such gregarious species is the tubicolous polychaete Phragmatopoma moerchi, which builds reefs of variable size on the rocky intertidal. These reefs provide refuge and food to many invertebrate species. The aim of this work is to characterize the macrofauna associated to these reefs and to determine the possible relationship between reef size (RS) and parameters of the associated community. Nine samples were taken from the intertidal at Cocholgüe ( $\left.36^{\circ} 35^{\prime} \mathrm{S}\right)$, between October and December, 2001. In the laboratory the samples were weighted, and the associated fauna was classified by taxa, and when possible, identified to species level. RS was then related with the following community descriptors: total number of individuals, species richness, diversity, dominance and evenness. Were found a total of 487 specimens of 43 taxa belonging to nine major groups. The taxa with more species present were Mollusca and Polychaeta (17 and 10, respectively). Direct and significant relationships were found between RS and some the community descriptors; diversity values were high but not significantly correlated with RS. The results of the present study demonstrate that these reefs shelter a dense and diverse invertebrate fauna, and therefore they are likely important for maintaining local biodiversity.

KEYwORDS: Rocky intertidal, macrofauna, biodiversity, Polychaeta, Phragmatopoma moerchi, reefs. 


\section{INTRODUCCION}

La zona intermareal alberga gran cantidad de especies que forman grandes agregaciones limitadas a ciertos niveles mareales. Ejemplos claros de esto son el extenso cinturón de mitílidos formado en la zona intermareal de Chile (Navarrete \& Castilla 1990), esponjas en la zona intermareal y submareal (Frith 1976), y las agregaciones de ascidias en fondos submareales someros e intermareales (Gutiérrez \& Lay 1965, Zamorano \& Moreno 1975, Fielding et al. 1994, Sepúlveda 2001).

Muchas de las especies de invertebrados que habitan estos tipos de agregaciones colonizan una variedad de microhábitats que pueden estar formados por algas y animales presentes en el intermareal rocoso (Frith 1976, Biernbaum 1981, Moore 1986, Tsuchiya \& Nishihira 1987, Costello \& Myers 1987, Tsuchiya \& Bellan-Santini 1989, Nelson \& Demetriades 1992, Krapp-Schickel 1993, Covi \& Kneib 1995, Conradi et al. 1997, Tena et al. 2000, Sepúlveda et al. 2003). En estos microhábitats los invertebrados pueden encontrar alimento y protección (Duffy \& Hay 1991, Schaffelke et al. 1995, Pavia et al. 1999, Thiel 1999, Sepúlveda et al. 2003). Así, organismos autotróficos como algas y heterótrofos como invertebrados, representan microhabitats esenciales para organismos que obtienen alimento directamente de ellos, o de sus epibiontes. Por otra parte, los sustratos que son construidos por organismos metazoos, pueden albergar altas densidades de invertebrados herbívoros que se alimentan de algas epífitas (e.g., Nelson \& Demetriades 1992). Otro grupo de invertebrados se alimenta del material que es transportado por las corrientes marinas hasta los lugares donde los animales están habitando. Estos animales pueden comprender organismos depositívoros o suspensívoros (Fenwick 1976, Krapp-Schickel 1993).

Estudios sobre epifauna asociada a Laminariales, esponjas y otros invertebrados como sustrato, indican una relación lineal entre el tamaño del sustrato biológico y el número de individuos y especies de la fauna asociada (Costello \& Myers 1987, Thiel \& Vásquez 2000). Dentro de estos microhábitats particulares se ha observado a menudo un aumento lineal en la abundancia de invertebrados (Villouta \& Santelices 1984, Thiel \& Vásquez 2000, Hernández et al. 2001, Sepúlveda et al. 2003). Sin embargo, entre algunos hábitats existen diferencias en el número de individuos por unidad de área (Vásquez \& Santelices 1984, Tsuchiya \& Nishihira 1985). Normalmente estas diferencias pueden relacionarse con algunas características del hábitat tales como la exposición a las olas, exposición a las corrientes, a la presión de depredación, o a la competencia con conespecíficos (Fenwick 1976, Edgar \& Aoki 1993, Sepúlveda 2001).

Entre los estudios de fauna asociada, los relacionados con algas y esponjas son los más numerosos (e.g., Frith 1976, Biernbaum 1981, Cancino \& Santelices 1981, Moore 1986, Buschmann 1990, López \& Stotz 1997, Boström \& Mattila 1999), mientras que los estudios de fauna asociada, utilizando poliquetos como sustrato, son escasos (e.g., Nelson \& Demetriades 1992, Zamorano 2000a).

Una de las especies de poliquetos capaz de formar agregaciones de individuos es el sabelárido tubícola Phragmatopoma moerchi Kinberg 1867, que al igual que otros sabeláridos (Insunza 1989) forma sus tubos cementando las aristas de diversos materiales, tales como silicatos terrígenos, trozos de concha, espinas de erizos, etc. (Anadon 1981), por medio de una sustancia mucosa insoluble en agua (Wilson 1970). Estas construcciones de material sobre rocas intermareales con influencia de abrasión de arena, forman arrecifes constituidos por un número variable de tubos, y por ende de individuos (Guíñez 1973), generando microhábitats que son utilizados por otras especies de invertebrados que aprovechan los intersticios entre los tubos para protegerse y alimentarse (Zamorano 2000b).

Es por esto que resulta de gran importancia (en términos ecológicos) determinar la fauna asociada a los arrecifes de P. moerchi en un sistema rocoso intermareal, relacionando algunos de los parámetros comunitarios de la fauna asociada en función del tamaño de los arrecifes.

\section{MATERIALES Y METODOS}

Entre los meses de octubre y diciembre de 2001 fueron extraídas aleatoriamente un total de nueve muestras desde el intermareal rocoso de playa Cocholgüe $\left(36^{\circ} 35^{\prime} \mathrm{S}\right)$ (Figura 1), las que 
Macroinvertebrados en arrecifes de P. moerchi. SEPúlVEDA, R. D. ET AL.

fueron depositadas en bolsas plásticas y transportadas al laboratorio para ser tamizadas en un cedazo geológico de $500 \mu \mathrm{m}$ de diámetro de malla. Los arrecifes fueron pesados sin organismos para determinar el tamaño de las muestras en gramos, preservándose todos los ejemplares en alcohol al $70 \%$ hasta su posterior determinación específica, ó al nivel taxonómico más bajo posible.

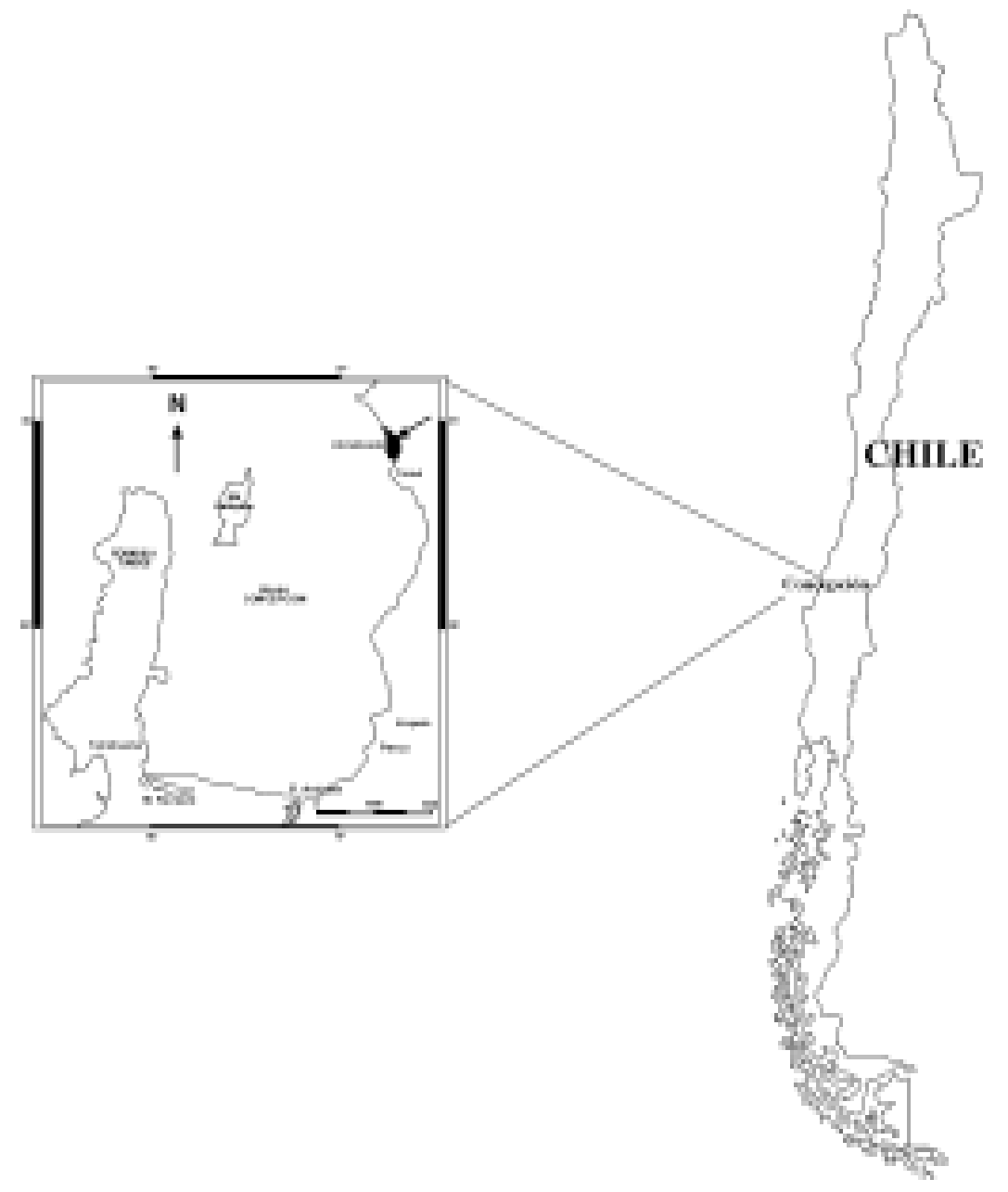

FIGURA 1. Ubicación geográfica de playa Cocholgüe en la Bahía Concepción.

FIGURE 1. Geographic location of Cocholgüe beach at Concepción Bay.

El análisis de la información se realizó mediante los índices de diversidad y uniformidad de Shannon-Wiener y el índice de dominancia de Simpson (Legendre \& Legendre 1979). En el cálculo de diversidad, se aplicó la técnica analítica de acuchillamiento de datos "Jackknife", que permite obtener estimadores paramétricos de la media, varianza y sesgo, posibilitando el cálculo de intervalos de confianza y dócima de hipótesis (Sokal \& Rohlf 1995).

Se aplicaron ajustes de regresión lineal para determinar la relación entre los componentes de diversidad y el tamaño de las muestras, y para determinar la relación del tipo rarefacción entre el número de especies y el número de individuos. 


\section{RESULTADOS}

Las variables diversidad y tamaño de las muestras, presentaron relaciones lineales directas entre sí. La relación entre la riqueza y el tamaño de las muestras mostró una relación no significativa $\left(\mathrm{R}^{2}=0.412\right.$;
$\left.\mathrm{F}_{(1,7)}=4.916 ; \mathrm{p}=0.062\right)$, mientras que la abundancia en función del tamaño de las muestras fue significativa $\left(\mathrm{R}^{2}=0.525 ; \mathrm{F}_{(1,7)}=7.747 ; \mathrm{p}=0.027\right.$ ) (Figura 2). La curva de rarefacción entre especies e individuos también mostró una relación significativa $\left(\mathrm{R}^{2}=\right.$ $0.900 ; F_{(1,7)}=63.676 ; p<0.01$ ) (Figura 3).

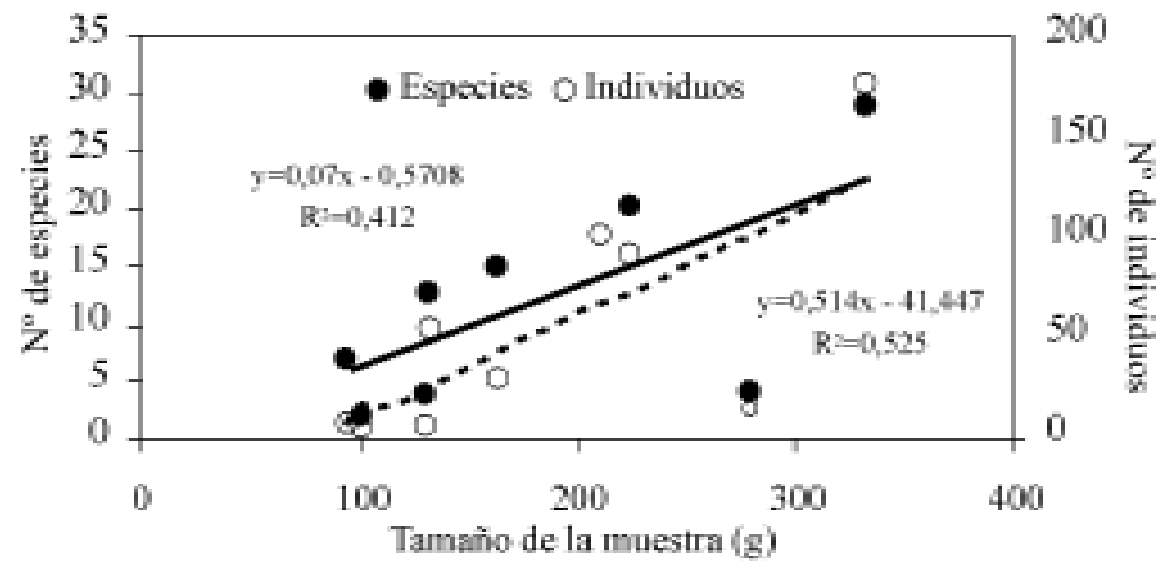

FIgURA 2. Relación de la riqueza (línea continua) y abundancia (línea punteada) en función del tamaño de las muestras. Ambas regresiones lineales, especies $(\mathrm{p}>0.05)$ e individuos $(\mathrm{p}<0.05)$.

FIGURE 2. Relationship between richness (solid line) and abundance (dashed line) as a function of sample size. Both linear regressions, species $(\mathrm{p}>0.05)$ and individuals $(\mathrm{p}<0.05)$.

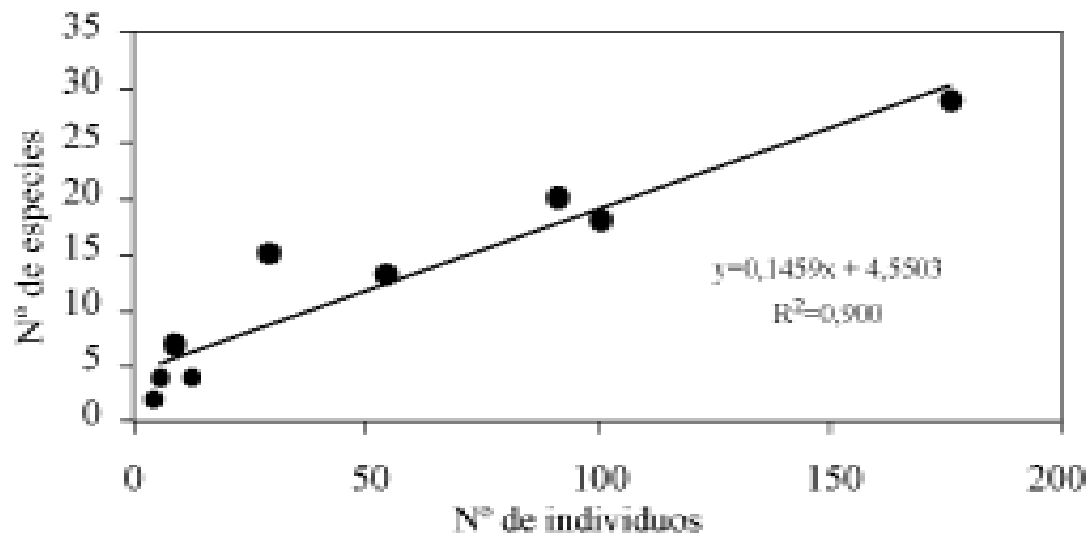

Figura 3. Relación entre el número de especies y el número de individuos. Regresión lineal y significativa $(\mathrm{p}<0.01)$.

FIGURE 3. Relationship between species number and individuals number. Lineal and significant regression $(\mathrm{p}<0.01)$. 


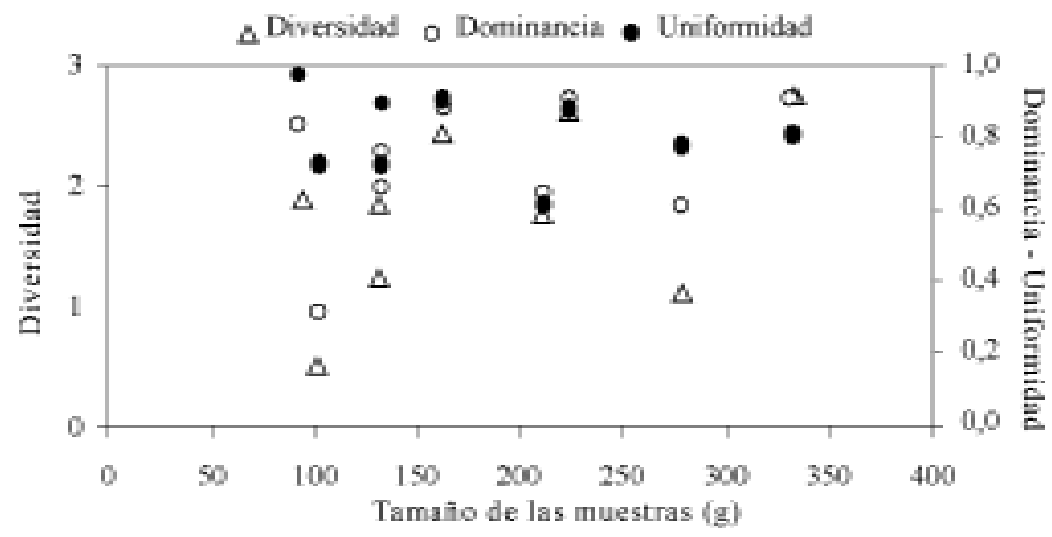

Figura 4. Valores de los índices de diversidad y de uniformidad de Shannon, y dominancia ecológica de Simpson en función del tamaño de las muestras. Ninguna relación es significativa ( $p>0.05)$.

FIGURE 4. Diversity and evenness Shannon indices values, Simpson's ecological dominance as a function of sample size. Neither relationship is significative $(\mathrm{p}>0.05)$.

Los valores de diversidad, dominancia y uniformidad fueron altos en todas las muestras, con excepción de la muestra tres, sin embargo no mostraron diferencias significativas $(\mathrm{p}>0.05)$ con respecto al tamaño de las muestras (Figura 4, Tabla I).

El análisis cuantitativo reveló un total de 487 especímenes distribuidos en 43 taxa, agrupados en nueve grupos mayores. Los grupos más diversos fueron Mollusca (17 especies) y Polychaeta (10 especies) (Tabla II). La mayor abundancia correspon- dió al poliqueto Typosyllis sp. $(21,97 \%$ del total analizado), seguido por Ischyromene menziesi (Sivertsen \& Holthuis, 1980) (7,39\%), Tegula atra (Lesson 1830) (7,19\%), Hyale maroubrae (Stebbing 1899) (6,98\%), Prisogaster niger (Wood $1828)(6,78 \%)$ y Nematoda indeterminados (6,57\%). Las especies más frecuentes fueron Tegula atra y Brachidontes granulata (Hanley 1843) con $77,78 \%$ y Nematoda indeterminados con $66,67 \%$ (Tabla II).

TABLA I. Valores de los índices de diversidad, dominancia y uniformidad de macroinvertebrados asociados a los arrecifes de Phragmatopoma moerchi en Cocholgüe, Chile. De estos índices, los valores de Jackknife (H’j), límite inferior (L1) y límite superior (L2) fueron calculados para todas las muestras.

TABLE I. Diversity, dominance and evenness indices values of macroinvertebrates associated to Phragmatopoma moerchi reefs at Cocholgüe, Chile. From those indices, jackknife's values (H'j), lower limit (L1) and upper limit (L2) were calculated for all samples.

\begin{tabular}{ccccc}
\hline Arrecife & Peso arrecife (g) & Diversidad & Dominancia Uniformidad \\
\hline 1 & 131,61 & 1,243 & 0,667 & 0,896 \\
2 & 93,95 & 1,887 & 0,840 & 0,970 \\
3 & 101,81 & 0,500 & 0,320 & 0,722 \\
4 & 280,39 & 1,091 & 0,604 & 0,787 \\
5 & 131,73 & 1,833 & 0,758 & 0,715 \\
6 & 164,01 & 2,448 & 0,889 & 0,904 \\
7 & 211,32 & 1,766 & 0,645 & 0,611 \\
8 & 333,34 & 2,749 & 0,913 & 0,816 \\
9 & 225,13 & 2,625 & 0,911 & 0,876 \\
\hline H5 & & 2,967 & 0,917 & 0,799 \\
H5 I.1 & & 2,914 & 0,907 & 0,737 \\
H5 L.2 & & 3,084 & 0,940 & 0,836 \\
\hline
\end{tabular}


Gayana 67(1), 2003

TABLA II. Lista de las especies presentes en los arrecifes de $P$. moerchi en Cocholgüe, Chile. Se presentan los valores de abundancia por muestra y total. *Señala taxa no cuantificado.

TABLE II. List of species present in Phragmatopoma moerchi reefs at Cocholgüe, Chile. Data include the values of abundance for each sample and for the total. *Indicates not quantified taxa.

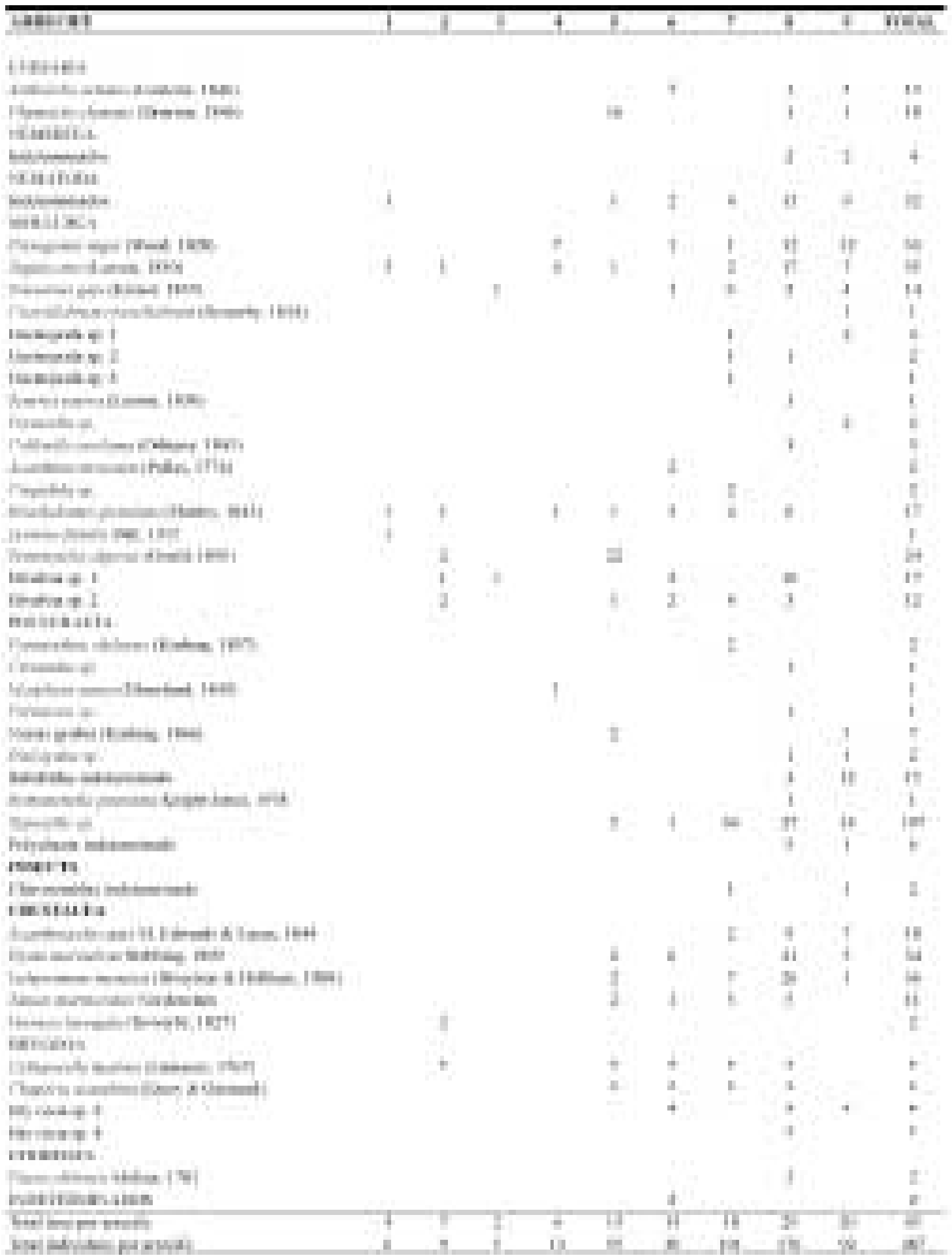




\section{DISCUSION}

Los arrecifes de $P$. moerchi albergan una alta diversidad de especies, y al igual que otros sistemas mareales sirven de albergue a pequeños invertebrados que se protegen del embate de las olas y de los depredadores (Navarrete \& Castilla 1990, Nelson \& Demetriades 1992, Zamorano 2000b, Sepúlveda et al. 2003). La ubicación de los arrecifes en el intermareal está determinada por la abrasión de arena y la exposición al oleaje, ya que su constitución estructural está dependiendo principalmente de la cantidad de arena disponible para crear los arrecifes (Pawlik 1988), además de un sustrato duro en donde las larvas puedan asentarse (Eckelbarger 1976, Pawlik 1988).

Los resultados de regresión de las variables de diversidad medidas son concordantes con los estudios realizados en discos de algas Laminariales (Santelices et al. 1980, Cancino \& Santelices 1981, Vásquez \& Santelices 1984, Villouta \& Santelices 1984, Thiel \& Vásquez 2000) y en ascidias solitarias (Zamorano \& Moreno 1975, Sepúlveda 2001, Sepúlveda et al. 2003).

Naturalmente, la creciente suma de individuos y el aumento del número de especies a medida que las muestras son extraídas en mayores tamaños o proporciones parece una situación repetida en muchos ambientes (e.g., Thiel \& Vásquez 2000, Sepúlveda et al. 2003).

La relación entre la riqueza y el tamaño de las muestras no concuerda con el modelo de equilibrio de MacArthur \& Wilson (1963), en donde la diversidad aumenta a medida que el tamaño del sustrato aumenta. En el presente estudio no parece estabilizarse debido a que el tamaño de las muestras extraídas parece ser muy pequeño, sugiriendo un potencial aumento por sobre el número de especies ya encontradas en arrecifes más grandes. Situación similar sucede en la relación número de individuos y tamaño de las muestras, donde se observa un aumento progresivo y lineal. Este aumento determinaría que la comunidad asociada es el resultado de una agregación de individuos, que van ingresando a los arrecifes a medida que se les ofrece un sustrato más grande.

Los resultados indican un claro dominio de poliquetos en la comunidad asociada a los arrecifes, lo que es coincidente con otros estudios realizados en comunidades bentónicas intermareales (Villouta
\& Santelices 1984, López \& Stotz 1997).

Debido a que el muestreo fue realizado en un período de reclutamiento, entre las especies asociadas a los arrecifes se observó gran cantidad de juveniles, situación que junto a las abundancias de organismos adultos podrían sostener varias hipótesis: 1) los arrecifes presentan una superficie adecuada para la fijación y asentamiento larval, funcionando como vivero natural para organismos invertebrados; 2) los arrecifes constituirían un hábitat transitorio para especies que habitan en las cercanías del mismo intermareal; y 3) los arrecifes actuarían como refugio constante contra la presión de depredación, ya que éste es uno de los procesos importantes en la regulación de la estructura comunitaria (Paine 1974, Menge 1976, Menge \& Sutherland 1976).

Por otro lado, la heterogeneidad de los arrecifes de $P$. moerchi puede proporcionar una gran variedad de microhábitats que brinden protección a los organismos frente a factores ambientales adversos. Dependiendo del tamaño de las irregularidades y de los organismos, podría ocurrir una posible emigración hacia otros ambientes, como lo sugiere la segunda hipótesis descrita más arriba. Similares observaciones han sido documentadas en macroinvertebrados asociados a macroalgas (Cancino \& Santelices 1984, Worthington \& Fairweather 1989, Moreno 1995, López \& Stotz 1997) y a mitílidos (Navarrete \& Castilla 1990).

Dada la asociación de macroinvertebrados que habitan temporalmente en los arrecifes de $P$. moerchi, concluimos que éstos albergarían una gran diversidad, comparada con otros hábitats de microescala similares (i.e. agregaciones de ascidias, grampones de algas Laminariales, parches de mitílidos y algas). Además, los arrecifes podrían funcionar como modelos de microescala en la conservación de la biodiversidad local.

\section{AGRADECIMIENTOS}

Los autores agradecen a P.A. Camus, J.M. Cancino y A.T. Palma por sus valiosas sugerencias en la revisión del manuscrito. R.D. Sepúlveda agradece en forma especial la beca de Magister CONICYT. 


\section{BIBLIOGRAFIA}

Anadon, N. 1981. Contribución al conocimiento de la fauna bentónica de la ría de Vigo III. Estudio de los arrecifes de Sabellaria alveolata (L.) (Polychaeta, Sedentaria). Investigación Pesquera 45:105-122.

Biernbaum, C. 1981. Seasonal changes in the amphipod fauna of Microciona prolifera (Ellis \& Solander) (Porifera: Demospongiae) and associated sponges in a shallow salt-marsh creek. Estuaries 4:85-96.

Boström, C. \& J. Mattila. 1999. The relative importance of food and shelter for seagrassassociated invertebrates: a latitudinal comparison of habitat choice by isopod grazers. Oecologia 120:162-170.

Buschmann, A. 1990. Intertidal macroalgae as refuge and food for Amphipoda in central Chile. Aquatic Botany 36:237-245.

Cancino, J.M. \& B. Santelices. 1981. The ecological importance of kelp-like holdfast as a habitat for invertebrates in central Chile. Proceedings International Seaweed Symposium 10:241246.

Cancino, J.M. \& B. Santelices. 1984. Importancia ecológica de los discos adhesivos de Lessonia nigrescens Bory (Phaeophyta) en Chile central. Revista Chilena de Historia Natural 57:23-33.

Conradi, M., P. López-González \& C. García-Gómez. 1997. The amphipod community as a bioindicator in Algeciras Bay (Southern Iberian Peninsula) based on a spatio-temporal distribution. Marine Ecology Progress Series 18:97-111

Costello, M. \& A. Myers. 1987. Amphipod fauna of the sponges Halichondria panicea and Hymeniacidon perleve in Lough Hyne, Ireland. Marine Ecology Progress Series 41:115-121.

Covi, M. \& R. KNeIB. 1995. Intertidal distribution, population dynamics and production of the amphipod Uhlorchestia spartinophila in a Georgia, USA, salt marsh. Marine Biology 121:447-455.

Duffy, J. \& M.E. Hay. 1991. Food and shelter as determinants of food choice by an herbivorous amphipod. Ecology 72:1286-1298.

Eckelbarger, K. 1976. Larval development and population aspects of the reef-building polychaete Phragmatopoma lapidosa from the east coast of Florida. Bulletin of Marine Science 26:117-132.

Edgar, G. \& M. AOKI. 1993. Resource limitation and fish predation: their importance to mobile epifauna associated with Japanese Sargassum. Oecologia 95:122-133.

Fenwick, G. 1976. The effect of wave exposure on the amphipod fauna of the alga Caulerpa brownii. Journal of Experimental Marine Biology and Ecology 25:1-18.

Fielding, P., K. Weerts \& A. Forbes. 1994. Macroinvertebrate communities associated with intertidal and subtidal beds of Pyura stolonifera (Heller) (Tunicata: Ascidiacea) on the Natal coast, South Africa. Journal of Zoology 29:46-51.

Frith, D. 1976. Animals associated with sponges at North Hayling Hampshire. Zoological Journal of the Linnean Society 58:353-362.

GuiÑEz, L. 1973. Contribución al conocimiento de algunos aspectos de la biología y ecología de Phragmatopoma moerchi Kinberg, 1867 (Annelida, Polychaeta, Sabellaridae) de caleta Leandro, Concepción, Chile. Tesis para optar al grado académico de Licenciado en Biología. Departamento de Biología Marina y Oceanografía. Universidad de Concepción. 290pp.

Gutiérrez, J. \& J. Lay. 1965. Observaciones biológicas en la población de Pyura chilensis Molina, 1782 en Antofagasta (Urochordata, Ascidiacea, Pyuridae). Estudios Oceanológicos 1:1-33.

Hernández, C., G. Muñoz \& N. Rozbaczylo. 2001. Poliquetos asociados con Austromegabalanus psittacus (Molina, 1782) (Crustacea: Cirripedia) en Península Gualpén, Chile central: Biodiversidad y efecto del tamaño del sustrato biológico. Revista de Biología Marina y Oceanografía 36:99-108.

InZUNZA, A. 1989. Granulometría y mineralogía en tubos de Phragmatopoma virgini (Polycheta, Sabellaridae) en el intermareal rocoso de Mehuín (Décima Región, Chile). Tesis para optar al título de profesor de estado en Biología y Química y Ciencias Naturales. Facultad de Filosofía y Humanidades. Universidad Austral de Chile. 45pp.

Krapp-Schickel, G. 1993. Do algal-dwelling amphipods react to the 'critical zones' of a coastal slope? Journal of Natural History 27:883-900.

Legendre, L. \& P. Legendre. 1979. Ecologie Numerique. V.2. La structure des données écologiques. Masson, Paris \& Presses de 1' Université du Quebec. 254pp.

López, C.A. \& W.B. Stotz. 1997. Descripción de la fauna asociada a Corallina officinalis $\mathrm{L}$. en el intermareal rocoso de la costa de "Palo Colorado" (Los Vilos, IV Región, Chile). Revista de Biología Marina y Oceanografía 32:17-35.

MacArthur, R.H. \& E.O. Wilson. 1963. An equilibrium theory of insular zoogeography. Evolution 17:373-387.

Menge, B.A. 1976. Organization of the New England rocky intertidal community: role of predation, competition and environmental heterogeneity. Ecological Monographs 46:355-393. 
Menge, B.A. \& J.P. Sutherland. 1976. Species diversity gradients: synthesis of the roles of predation, competition and temporal heterogeneity. American Naturalist 110:351-369.

Moore, P. 1986. Seaweed-associated animal communities in the Firth of Clyde, with special reference to the population biology of the amphipod Hyale nilssoni (Rathke). Proceedings of the Royal Society of Edinburgh 90B:271-286.

Moreno, C.A. 1995. Macroalgae as a refuge from predation for recruits of the mussel Choromytilus chorus (Molina, 1782) in Southern Chile. Journal of Experimental Marine Biology and Ecology 191:181-193.

Navarrete, S.A. \& J.C. Castilla. 1990. Resource partitioning between intertidal predatory crabs: interference and refuge utilization. Journal of Experimental Marine Biology and Ecology 143:101-129.

Nelson, W. \& L. Demetriades. 1992. Peracarids associated with sabellariid worm rock (Phragmatopoma lapidosa Kinberg) at Sebastian Inlet, Florida, U.S.A. Journal of Crustacean Biology 12:647-654.

PaINE, R.T. 1974. Intertidal community structure. Experimental studies on the relationship between a dominant competidor and its principal predator. Oecologia 15:93-120.

Pavia, H., H. Carr \& P. Åberg. 1999. Habitat and feeding preferences of crustacean mesoherbivores inhabiting the brown seaweed Ascophyllum nodosum (L.) Le Jol. and its epiphytic macroalgae. Journal of Experimental Marine Biology and Ecology 236:1532.

PAWLIK, J. 1988. Larval settlement and metamorphosis of sabellariid polychaetes, with special reference to Phragmatopoma lapidosa, a reefbuilding species, and Sabellaria floridensis, a non-gregarious species. Bulletin of Marine Science 43:41-60.

Santelices, B., J.C. Castilla, J.M. Cancino \& P. Schmiede. 1980. Comparative ecology of Lessonia nigrescens and Durvillaea antarctica (Phaeophyta) in central Chile. Marine Biology 59:119-132.

Schaffelke, B., D. Evers \& A. Walhorn. 1995. Selective grazing of the isopod Idotea baltica between Fucus evanescens and F. vesiculosus from Kiel Fjord (western Baltic). Marine Biology 124:215-218.

SepúlvedA, R.D. 2001. Diversidad de la macrofauna de invertebrados asociados a las agregaciones de Pyura chilensis Molina, 1782 (Tunicata: Ascidiacea). Tesis de Licenciatura y Título Biología Marina. Facultad de Ciencias, U. Católica de la Santísima Concepción, 60pp.

Sepúlveda, R.D., J.M. Cancino \& M. Thiel. 2003. The peracarid epifauna associated with the ascidian Pyura chilensis Molina, 1782 (Ascidiacea: Pyuridae). Journal of Natural History 37: 1555-1569.

Sokal, R. \& F. Rohlf. 1995. The principles and practice of statistics in biological research. Third edition. W. H. Freeman and Company, New York. 887pp.

Tena, J., R. Cappaccioni, F. Torres \& A. García. 2000. Polychaetes associated with different facies of the photophilic algal community in the Chafarinas archipelago (SW Mediterranean). Bulletin of Marine Science 67:55-72.

Thiel, M. 1999. Host-use and population demographics of the ascidian-dwelling amphipod Leucothoe spinicarpa: indication for extended parental care and advanced social behaviour. Journal of Natural History 33:193-206.

Thiel, M. \& J.A. VÁsquez. 2000. Are kelp holdfasts islands on the ocean floor? Indication for temporarily closed demes of peracarid crustaceans. Hydrobiologia 440:45-54.

Tsuchiya, M. \& D. Bellan-Santini. 1989. Vertical distribution of shallow rocky shore organisms and community structure of mussel beds (Mytilus galloprovincialis) along the coast of Marseille, France. Mésogée 49:91-110.

TsuchiYa, M. \& M. Nishinira. 1985. Islands of Mytilus edulis as a habitat for small intertidal animals: effects of island size on community structure. Marine Ecology Progress Series 25:71-81.

TsuchiYA, M. \& M. Nishinira. 1987. Islands of Mytilus edulis as a habitat for small intertidal animals: effects of Mytilus age structure on the species composition of the associated fauna and community organization. Marine Ecology Progress Series 31:171-178.

VÁsquez, J.A. \& B. Santelices. 1984. Comunidades de macroinvertebrados en discos adhesivos de Lessonia nigrescens Bory (Phaeophyta) en Chile central. Revista Chilena de Historia Natural 57:131-154.

Villouta, E. \& B. Santelices. 1984. Estructura de la comunidad submareal de Lessonia (Phaeophyta, Laminariales) en Chile norte y central. Revista Chilena de Historia Natural 57:111122.

Wilson, D.P. 1970. Additional observations on larval growth and settlement of Sabellaria alveolata (L.). Journal Marine Biological Association of the U.K. 50:1-31.

Worthington, D. \& P.G. Fairweather. 1989. Shelter and food: interactions between Turbo undulatum (Archaeogastropoda: Turbinidae) and coralline algae on rocky seashores in New South Wales. Journal of Experimental Marine Biology and Ecology 129:61-79.

Zamorano, J.H. 2000a. Fauna asociada a las agregaciones del poliqueto tubícola Phragmatopoma virgini Kinberg, en el intermareal rocoso de Mehuín. XX Congreso de Ciencias 
Gayana 67(1), 2003

del Mar, Concepción. 217.

Zamorano, J.H. 2000b. Efecto de la depredación sobre la distribución de Phragmatopoma moerchi Kinberg, 1867 (Polychaeta: Sabellariidae), en el intermareal rocoso de Mehuín, Sur de Chile. Tesis de Magister en Ciencias, Universidad
Austral de Chile, Valdivia. 125pp.

Zamorano, J.H. \& C.A. Moreno. 1975. Comunidades bentónicas del sublitoral rocoso de Bahía Corral. I. Area mínima de muestreo y descripción cuantitativa de la asociación de Pyura chilensis Molina. Medio Ambiente 1:58-66.

Fecha de recepción: 04/11/02

Fecha de aceptación: 23/01/03 